The Journal of Snmolic Logic

Volume 34, Number 4, Dec. 1969

\title{
AN ABSTRACT FORM OF THE CHURCH-ROSSER THEOREM. I
}

\section{R. HINDLEY}

One of the basic results in the theory of $\lambda$-conversion is the Church-Rosser Theorem, which says that, using certain rules for conversion and reduction of $\lambda$-formulae, any two interconvertible formulae can both be reduced to one formula. (I will not explain this in detail, as $\lambda$-conversion is described fully in Church's [2], where the Church-Rosser Theorem is Theorem 7 XXVII; see also Chapter 4 of Curry and Feys' [3].) The first part of the present paper contains an abstract form of this theorem.

In the second part the abstract result will be applied to prove the Church-Rosser Theorem for $\lambda$-reduction, and for any reduction defined as a series of replacements of parts of formulae (satisfying certain conditions); this covers Church's $\delta$-reduction, and Curry's weak reduction in combinatory logic (with or without extra arithmetical combinators, and with or without type-restrictions), but does not cover strong reduction $[3, \S 6 \mathrm{~F}]$ or Curry's $\lambda-\eta$-reduction $[3, \S 4 \mathrm{D}] .^{1}$

At the end of Part II a simple abstract theorem will be proved which extends the Church-Rosser Theorem to include $\lambda-\eta$-reduction; this seems to be simpler than the extension proof in [3, Chapter 4].

Abstract forms of the Church-Rosser Theorem have already been proved by Newman in [4] and Curry in [1], but Rosser and Schroer pointed out in [5] that they do not cover the original theorem as a special case. In [6], Schroer has proved an abstract result which does cover the original one; however his methods are quite different from those used here, and I do not think his result covers, or is covered by, the result in the present paper. Part of Curry's proof of the Church-Rosser Theorem in [3, Chapter 4] is in an abstract setting, though the assumptions he uses are slightly more restrictive than the ones used here.

I am very grateful to $R$. Harrop for his help and supervision during the work for this paper (which is part of my thesis at the University of Newcastle upon Tyne), and to the Northern Ireland Ministry of Education, who financed it through a Research Studentship.

§1. Primitive ideas. Most of the following notation is from Curry's [1], with some from Schroer [6].

There is assumed to be a set of objects called points; these may be interpreted as $\lambda$-formulae (more precisely, as equivalence classes with respect to change of bound variable), and they will be denoted by capital letters.

There are also certain cells; to each cell is associated two points, its start and its

Received May 16, 1967.

1 Part II is to be published later.

1一J.S.L. 
terminus, and the cell is said to be from its start to its terminus. Cells will be denoted by " $\xi$ ", " $\eta$ ", " $\zeta$ ", and in $\lambda$-conversion a cell is a single contraction $[2$, p. 14].

Every set of cells considered here will be coinitial; that is, all its members have the same start; " $\alpha$ ", " $\beta$ " will denote such sets, and " $\left\{\xi_{1}, \cdots, \xi_{n}\right\}$ " will denote the set whose only members are $\xi_{1}, \cdots, \xi_{n}$, and the empty set $\varnothing$ when $n=0$. Unless stated otherwise it will be assumed that in a set $\left\{\xi_{1}, \cdots, \xi_{n}\right\}$ members with distinct indices are distinct. One-member sets will not be distinguished from their sole members. For any sets $\alpha_{1}, \cdots, \alpha_{n}$ of cells, all coinitial, " $\left\{\alpha_{1}, \cdots, \alpha_{n}\right\}$ " will denote the union of $\alpha_{1}, \cdots, \alpha_{n}$, and will be empty when $n=0$.

Instead of the relation $J$ in [1], I shall assume that a binary relation $\prec$ has been defined to hold between certain pairs of coinitial cells. (In $\lambda$-conversion if $\xi$ and $\eta$ are cells starting at $X$, and $\xi$ is the contraction of a part $P$ of $X$, and $\eta$ is the contraction of $Q$, then $\xi<\eta$ will mean that $P$ is part of $Q$.) " $\xi \nless \eta$ " is defined to mean that $\xi$ and $\eta$ are coinitial and not $\xi \prec \eta$.

The derivative, $\xi / \eta$, of $\xi$ with respect to $\eta$, is a set assumed to be associated with each ordered pair $\xi, \eta$ of coinitial cells. If it is not empty, its members (the residuals of $\xi$ with respect to $\eta$ ) are assumed to be cells starting at the terminus of $\eta$. (For $\lambda$-conversion, residuals are defined in $[2$, p. 18].)

A reduction from $A$ to $B$ is a sequence $\xi_{1}, \cdots, \xi_{m}$ of cells (called steps of the reduction) such that $\xi_{1}$ starts at $A$, each $\xi_{i+1}$ starts at the terminus of $\xi_{i}$, and $\xi_{n}$ terminates at $B . A$ and $B$ are called the start and terminus of the reduction and $m$ is the length of the reduction. Letters " $\rho "$, " $\sigma$ ", " $\tau$ " will denote reductions; if $m=1$, the reduction will not be distinguished from its one step. For each point $A$ there is a null reduction from $A$ to $A$, with length 0 ; all null reductions will be called " 0 ".

The sum, $\rho+\sigma$, of a reduction $\rho$ and a reduction $\sigma$ which starts at the terminus of $\rho$, is the result of putting the steps of $\sigma$ in order after the steps of $\rho$; also $\rho+0$ and $0+\rho$ are defined to be $\rho$.

The derivative, $\xi / \rho$, of $\xi$ with respect to a reduction $\rho$ coinitial with $\xi$, is defined by induction thus:

$$
\begin{aligned}
\xi / 0 & =\xi, \\
\xi /(\rho+\eta) & =\text { union of all } \xi^{\prime} / \eta \text { for } \xi^{\prime} \text { in } \xi / \rho .
\end{aligned}
$$

For a set $\alpha, \alpha / \rho$ is the union of all $\xi / \rho$ for all $\xi$ in $\alpha$.

Notice the following properties of sums and derivatives:

$$
\begin{aligned}
\rho+(\sigma+\tau) & =(\rho+\sigma)+\tau, \\
\alpha /(\rho+\sigma) & =(\alpha / \rho) / \sigma, \\
\varnothing / \rho & =\varnothing .
\end{aligned}
$$

$A$ development of $a$ set $\alpha$ is a reduction $\xi_{1}, \ldots, \xi_{n}$ such that $\xi_{1}$ is in $\alpha$ and for each $i$,

$$
\xi_{i+1} \text { is in } \alpha /\left(\xi_{1}+\cdots+\xi_{i}\right) .
$$

Such a development is complete just when

$$
\alpha /\left(\xi_{1}+\cdots+\xi_{n}\right)=\varnothing .
$$

It can be seen that if $\rho$ is a development of $\alpha$, and $\sigma$ starts at the terminus of $\rho$, then $\rho+\sigma$ is a (complete) development of $\alpha$ if and only if $\sigma$ is a (complete) development of $\alpha / p$. 
For reductions $\rho$ and $\sigma$, the equivalence

$$
\rho \simeq \sigma
$$

is defined to mean that $\rho$ has the same start and terminus as $\sigma$. Then for all $\tau_{1}$ and $\tau_{2}$ :

$$
\rho \simeq \sigma \Rightarrow \rho+\tau_{1} \simeq \sigma+\tau_{1} \text { and } \tau_{2}+\rho \simeq \tau_{2}+\sigma .
$$

Two points $A$ and $B$ are connected if there is a sequence $A_{0}, \cdots, A_{n}$ of points such that $A_{0}=A, A_{n}=B$, and for each $i$ there is a cell from $A_{t-1}$ to $A_{2}$ or from $A_{1}$ to $A_{i-1}$. For example in Figure $1, A_{0}$ and $A_{5}$ are connected, there being cells from $A_{0}$ to $A_{1}, A_{1}$ to $A_{2}, A_{3}$ to $A_{2}$, and so on.

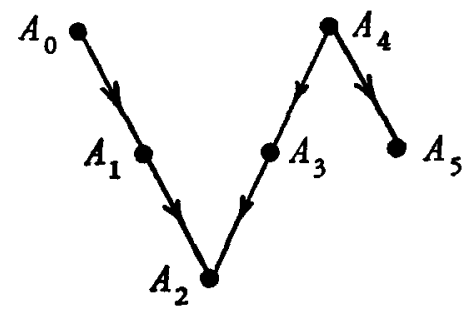

FIGURE 1

One of the main tools used in this paper is the concept of Minimal Complete Development ${ }^{2}$ which will now be defined; it is very like a concept used by Curry and Feys in part of their proof of the Church-Rosser Theorem in [3, $84 \mathrm{C} 3]$. A cell $\xi$ is minimal in a set $\beta$ of cells if $\xi \in \beta$ and

$$
\eta \in \beta \text { and } \eta \neq \xi \Rightarrow \eta K \xi \text {. }
$$

A complete development, $\xi_{1}+\cdots+\xi_{n}$, of a set $\alpha$ of cells is an $M C D$ of $\alpha$ if for each $i, \xi_{1+1}$ is minimal in $\alpha /\left(\xi_{1}+\cdots+\xi_{i}\right)$.

Finally, " $\xi / \zeta K \eta / \zeta$ " is defined to mean that there do not exist $\xi$ ' in $\xi / \zeta$ and $\eta$ ' in $\eta / \zeta$ such that $\xi^{\prime} \prec \eta^{\prime}$, so in particular it is true if either $\xi / \zeta$ or $\eta / \zeta$ is empty.

\$2. Main theorem. The Church-Rosser property is the abstract analogue of the conclusion of the Church-Rosser Theorem; it says that for any connected points $A$ and $B$, there exist reductions $\rho$ and $\sigma$ starting at $A$ and $B$ respectively, with a common terminus. (See Figure 2.)



FIGURE 2

" Called "MCD" for short. 
This property can fairly easily [3, Chapter 4, Theorems 3 and 4] be proved equivalent to the following:

(C) If a reduction $\rho$ and $a$ cell $\xi$ are coinitial, then there exist reductions $\sigma$ and $\tau$ such that $\rho+\sigma \simeq \xi+\tau$. (See Figure 3.)

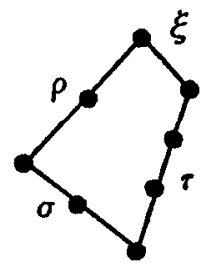

Figure 3

THEOREM 1. Property (C) is implied by the conjunction of the following eight assumptions:

(A1). $\xi \prec \eta \Rightarrow \eta K \xi$.

(A2). $\xi \prec \eta \& \eta \prec \zeta \Rightarrow \xi \prec \zeta$.

(A3). If $\xi K \eta$, then $\xi / \eta$ has no more than one member.

(A4). $\xi / \xi=\varnothing$.

(A5). $\eta_{1} \nless \xi$ and $\eta_{1} K \eta_{2} \Rightarrow \eta_{1} / \xi \nless \eta_{2} / \xi$.

(A6). If $\eta_{i}<\xi$ for $i=1, \cdots, n$, then there exists $k$ such that for all $j \neq k$, $\eta_{j} K \eta_{k}$ and $\eta_{j} / \xi K \eta_{k} / \xi$.

(A7). If $\xi$ and $\eta$ are coinitial, then there exist an $M C D \rho$ of $\xi / \eta$ and an $M C D \sigma$ of $\eta / \xi$, such that $\xi+\sigma \simeq \eta+\rho$.

(A8). If (A7) is true and $\zeta$ is any cell coinitial with $\xi$ and $\eta$, then $\zeta /(\xi+\sigma)=$ $\zeta /(\eta+\rho)$ in the following two cases:

(i) $\zeta \nless \xi$ and $\zeta K \eta$,

(ii) $\eta \prec \xi$ and $\zeta \prec \xi$ and $\zeta K \eta$ and $\zeta / \xi K \eta / \xi$.

\$3. Proof of the main theorem. For any coinitial cells $\xi$ and $\eta, \xi / / \eta$ is defined to be the MCD $\rho$ whose existence is assumed in (A7). If $\xi / \eta$ has just one member, then that member will be called " $\xi / \eta$ ", and so using this convention, $\xi / \eta=\xi / \eta$ in this case.

Before $(C)$ is derived, the following Lemmas 1-8 will be deduced from (A1), $\cdots$, (A8): first notice that by (A1), $\xi \nless \xi$ for all $\xi$.

LEMMA 1. (C) is implied by the following property:

$\left(C_{1}\right)$. If a cell $\xi$ and an $M C D \rho$ are coinitial, then there exist $M C D s \tau$ and $\sigma$ such that $\rho+\sigma \simeq \xi+\tau$.

The proof is by induction on the length of $\rho$, using in the basis the fact that any single cell is an MCD of the set consisting of itself alone, by (A4).

LEMMA 2. Any finite nonempty set of coinitial cells has a minimal member (by (A2) and (A1)).

LEMMA 3. By (A1), $\cdots$, (A4), every finite set of cells has an $M C D$; in fact, to each minimal member of the set corresponds an MCD whose first cell is that member.

The proof is by induction on the number of members in the set, using Lemma 2. 
LEMMA 4. To every MCD $\rho$ corresponds a finite set $\alpha$ of cells, of which $\rho$ is an $M C D$.

Proof. If $\rho$ is an MCD of $\beta$, take $\alpha$ to be the set of all the cells in $\beta$ whose derivatives actually occur in $\rho$.

LEMMA 5. If $\rho$ is a development of $\alpha$, and $\xi K \eta$ for all $\eta \in \alpha$, then $\xi / \rho$ has at most one member, and $\xi / \rho K \eta / \rho$ for all $\eta \in \alpha$.

The proof is by induction on the length of $\rho$, using (A5) and (A3).

LEMMA 6. If $\eta_{1}, \cdots, \eta_{m}, \xi_{1}, \cdots, \xi_{n}$ are mutually coinitial, $\rho$ is an MCD of $\left\{\eta_{1}, \cdots, \eta_{m}\right\}$ and $\xi_{i} K \eta_{j}$ for all $i=1, \cdots, m$ and $j=1, \cdots, n$, then

$$
\rho+\text { any } M C D \text { of }\left\{\xi_{1} / \rho, \cdots, \xi_{x} / \rho\right\}
$$

is an $M C D$ of $\left\{\eta_{1}, \cdots, \eta_{m}, \xi_{1}, \cdots, \xi_{n}\right\}$.

The proof is by induction on $n$, using Lemma 5 .

LEMMA 7. If $\xi_{1}, \cdots, \xi_{m}$ are mutually coinitial, and

$$
j>i \Rightarrow \xi_{j} K \xi_{i}
$$

then the reduction $\zeta_{1}+\cdots+\zeta_{t}$ defined by letting $\zeta_{k+1}$ be the (sole) member of the first of $\left(\xi_{1} /\left(\zeta_{1}+\cdots+\zeta_{k}\right)\right), \cdots,\left(\xi_{m} /\left(\zeta_{1}+\cdots+\zeta_{k}\right)\right)$ which is not empty, is an $M C D$ of $\left\{\xi_{1}, \cdots, \xi_{m}\right\}^{3}$

The proof is by Lemma 6 (with $n=1$ ), used at most $m$ times.

LEMMA 8. If $\rho$ and $\sigma$ are $M C D$ s of $\left\{\eta_{1}, \cdots, \eta_{n}\right\}$, and $\xi K \eta_{i}$ for $i=1, \cdots, n$, then

$$
\rho \simeq \sigma \text { and } \xi / \rho=\xi / \sigma \text {. }
$$

Proof. Induction on $\boldsymbol{n}$ is used.

When $n=0$ : the only possible MCD is null.

When $n=1:$ the only MCD is $\eta_{1}$ itself.

When $n>1: \eta_{1}, \cdots, \eta_{n}$ can be relabelled so that the first cell of $\rho$ is $\eta_{1}$.

Then $\rho=\eta_{1}+\rho^{\prime}$, where $\rho^{\prime}$ is an MCD of the fewer-than- $n$ cells in $\left\{\eta_{2} / \eta_{1}, \cdots, \eta_{n} / \eta_{1}\right\}$. If $\sigma$ starts with the same cell as $\rho$, then $\sigma=\eta_{1}+\sigma^{\prime}$, where $\sigma^{\prime}$ is an MCD of $\left\{\eta_{2} / \eta_{1}, \cdots, \eta_{n} / \eta_{1}\right\}$. By the induction-hypothesis, $\rho^{\prime} \simeq \sigma^{\prime}$, and hence $\rho \simeq \sigma$. Also $\xi / \eta_{1}$ has at most one member, $\xi^{\prime}$, and by (A5), $\xi^{\prime} K \eta_{2} / \eta_{1}$ for each $i=2, \cdots, n$. So again by the induction-hypothesis, $\xi^{\prime} / \rho^{\prime}=\xi^{\prime} / \sigma^{\prime}$ and therefore $\xi / \rho=\xi / \sigma$.

Now suppose that the first cell of $\sigma$ is not $\eta_{1}$; then $\eta_{2}, \cdots, \eta_{n}$ can be relabelled so that the first cell of $\sigma$ is $\eta_{2}$, and so $\sigma=\eta_{2}+\sigma^{\prime}$, where $\sigma^{\prime}$ is an MCD of the fewerthan- $n$ cells in $\left\{\eta_{1} / \eta_{2}, \cdots, \eta_{n} / \eta_{2}\right\}$. The induction-step now breaks into two stages.

Stage 1. By definition of $\rho$ and $\sigma$ as MCDs, $\eta_{i} K \eta_{1}$ for all $i \neq 1$, and $\eta_{3} K \eta_{2}$ for all $j \neq 2$. Therefore by (A5), for $i=3, \cdots, n$.

$$
\eta_{i} / \eta_{1} \nless \eta_{2} / \eta_{1} \text {. }
$$

If $\eta_{2} / \eta_{1}$ is not empty, then it must have at most one member, by (A3), and by above and Lemma 3 there exists an MCD of $\left\{\eta_{2} / \eta_{1}, \cdots, \eta_{n} / \eta_{1}\right\}$ whose first cell is $\eta_{2} / \eta_{1}$. (See Figure 4.) Suppose this MCD is $\left(\eta_{2} / / \eta_{1}\right)+\rho^{\prime \prime}$; then $\rho^{\prime \prime}$ is an MCD of

$$
\left\{\left(\eta_{3} / \eta_{1}\right) /\left(\eta_{2} / / \eta_{1}\right), \cdots,\left(\eta_{n} / \eta_{1}\right) /\left(\eta_{2} / / \eta_{1}\right)\right\}
$$

which is the same, by definition, as

$$
\left\{\eta_{3} /\left(\eta_{1}+\eta_{2} / / \eta_{1}\right), \cdots, \eta_{n} /\left(\eta_{1}+\eta_{2} / / \eta_{1}\right)\right\}
$$

${ }^{3} \zeta_{0}$ is defined to be $\xi_{0}$. 
By the induction-hypothesis, $\rho^{\prime} \simeq\left(\eta_{2} / / \eta_{1}\right)+\rho^{\prime \prime}$, because both reductions are MCDs of $\left\{\eta_{2} / \eta_{1}, \cdots, \eta_{n} / \eta_{1}\right\}$. If $\eta_{2} / \eta_{1}$ is empty, define $\rho "$ to be $\rho^{\prime}$. Then $\left(\eta_{2} / / \eta_{1}\right)$ $+\rho^{\prime \prime}=0+\rho^{\prime \prime}=\rho^{\prime}$.

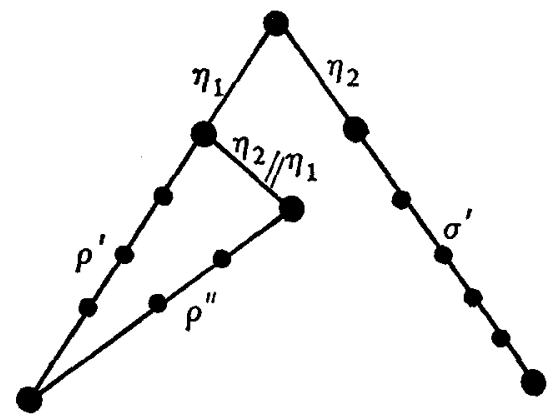

Figure 4

Residuals of $\xi$. If $\xi=\eta_{i}$ for some $i$, then $\xi / \sigma=\xi / \rho=0$ by definition of complete development. From now on, assume that $\xi \neq \eta_{i}$ for $i=1, \cdots, n$. By (A3), $\xi / \eta_{1}$ either is empty or contains only one cell, $\xi^{\prime}$. If $\xi / \eta_{1}$ is empty, then $\xi / \rho$ and $\xi /\left(\eta_{1}+\left(\eta_{2} / / \eta_{1}\right)+\rho^{\prime \prime}\right)$ are both empty. If $\xi / \eta_{1}$ contains only $\xi^{\prime}$, then by (A5), $\xi^{\prime} K \eta_{i} / \eta_{1}$ for $i=2, \cdots, n$. Hence $\xi^{\prime} / \rho^{\prime}=\xi^{\prime} /\left(\left(\eta_{2} / / \eta_{1}\right)+\rho^{\prime \prime}\right)$ by the inductionhypothesis applied to $\rho^{\prime},\left(\eta_{2} / / \eta_{1}\right)+\rho^{\prime \prime}, \xi^{\prime}$. Also, by (A3) $\xi^{\prime} /\left(\eta_{2} / / \eta_{1}\right)$ has at most one member. Therefore

$$
\xi / \rho=\xi /\left(\eta_{1}+\rho^{\prime}\right)=\xi^{\prime} / \rho^{\prime}=\xi^{\prime} /\left(\left(\eta_{2} / / \eta_{1}\right)+\rho^{\prime \prime}\right)=\xi /\left(\eta_{1}+\left(\eta_{2} / / \eta_{1}\right)+\rho^{\prime}\right) .
$$

Summarizing: whether $\eta_{2} / \eta_{1}$ is empty or not, $\left(\eta_{1}+\left(\eta_{2} / / \eta_{1}\right)+\rho\right)$ is an MCD of $\left\{\eta_{1}, \cdots, \eta_{n}\right\}$ with the same terminus as $\rho$, and the derivatives of $\xi$ are the same with respect to both reductions. Similarly, if $\sigma^{\prime \prime}$ is an MCD of

$$
\left\{\eta_{3} /\left(\eta_{2}+\eta_{1} / / \eta_{2}\right), \cdots, \eta_{n} /\left(\eta_{2}+\eta_{1} / / \eta_{2}\right)\right\}
$$

then $\left(\eta_{2}+\left(\eta_{1} / / \eta_{2}\right)+\sigma^{\prime \prime}\right)$ is an MCD of $\left\{\eta_{1}, \cdots, \eta_{n}\right\}$ with the same terminus and derivative of $\xi$ as $\sigma$ has. (See Figure 5.)

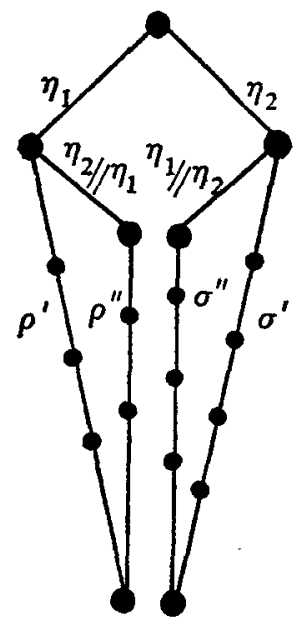

FIGURE 5 
Stage 2. By (A7), $\eta_{1}+\left(\eta_{2} / / \eta_{1}\right) \simeq \eta_{2}+\left(\eta_{1} / / \eta_{2}\right)$ and by (A8) part (i) the derivatives of $\xi$ and of $\eta_{3}, \cdots, \eta_{n}$ are the same with respect to either reduction (from Stage 1, each derivative can be seen to have at most one member). Hence applying the induction-hypothesis to $\rho^{\prime \prime}, \sigma^{\prime \prime}$ and $\xi /\left(\eta_{1}+\eta_{2} / / \eta_{1}\right)$ (which is the same as $\left.\xi /\left(\eta_{2}+\eta_{1} / / \eta_{2}\right)\right)$ shows that $\rho^{\prime \prime} \simeq \sigma^{\prime \prime}$ and

$$
\xi /\left(\eta_{1}+\left(\eta_{2} / / \eta_{1}\right)+\rho^{\prime \prime}\right)=\xi /\left(\eta_{1}+\left(\eta_{2} / / \eta_{1}\right)+\sigma^{\prime \prime}\right)=\xi /\left(\eta_{2}+\left(\eta_{1} / / \eta_{2}\right)+\sigma^{\prime \prime}\right) .
$$

Therefore, using Stage 1,

$$
\rho \simeq\left(\eta_{1}+\left(\eta_{2} / / \eta_{1}\right)+\rho^{\prime \prime}\right) \simeq\left(\eta_{1}+\left(\eta_{2} / / \eta_{1}\right)+\sigma^{\prime \prime}\right) \simeq\left(\eta_{2}+\left(\eta_{1} / / \eta_{2}\right)+\sigma^{\prime \prime}\right) \simeq \sigma,
$$

and $\xi / \rho=\xi / \sigma$, as required.

DefinItion 1. For any mutually coinitial cells $\xi, \eta_{1}, \cdots, \eta_{n}$, a $\xi$-MCD of $\left\{\eta_{1}, \cdots, \eta_{n}\right\}$ is a minimal complete reduction constructed according to the following rules. ${ }^{4}$

If $n=0$, define the $\xi$-MCD to be 0 . Otherwise, first renumber the cells $\eta_{1}, \cdots, \eta_{n}$ so that for some number $m$ :

for $i=1, \cdots, m, \xi K \eta_{l}$ and $\xi \neq \eta_{i}$; (if there are no such $\eta_{i}$, set $m=0$ ),

for $i=(m+1), \cdots, n, \xi \prec \eta_{i}$ or $\xi=\eta_{i}$.

Also, using Lemma 2, number $\eta_{m+1}, \cdots, \eta_{n}$ so that

$$
h>j \Rightarrow \eta_{m+h} K \eta_{m+j}
$$

and if $\xi=\eta_{m+k}$ for some $k$, arrange the cells so that $k=1$. (This is possible because by (A1) and the definition of $m, \xi$ would be minimal in $\left\{\eta_{m+1}, \cdots, \eta_{n}\right\}$.) Notice that $\eta_{m+1} K \eta_{1}$ for $i=1, \cdots, m$ and $j=1, \cdots,(n-m)$ because otherwise, either $\xi \prec \eta_{m+j} \prec \eta_{i}$ or $\xi=\eta_{m+j} \prec \eta_{i}$, which both imply $\xi \prec \eta_{l}$, contrary to the definition of $i$. Hence by Lemma 6 , if $\rho$ is any MCD of $\left\{\eta_{1}, \cdots, \eta_{m}\right\}$ and $\sigma$ is any MCD of $\left\{\eta_{m+1} / \rho, \cdots, \eta_{n} / \rho\right\}$, then $\rho+\sigma$ will be an MCD of $\left\{\eta_{1}, \cdots, \eta_{n}\right\}$. To define a $\xi-\mathrm{MCD}$, it remains to give rules for constructing $\rho$ and $\sigma$.

Define $\rho$ to be the last member of a sequence $\rho_{0}, \rho_{1}, \cdots$ of reductions constructed as follows. Choose $\rho_{0}=0$. To construct $\rho_{k+1}$, notice first that if $\rho_{k}$ is constructed and is part of an MCD of $\left\{\eta_{1}, \cdots, \eta_{m}\right\}$, then by Lemma $5, \xi / \rho_{k} K \eta_{k} / \rho_{k}$ for $i=$ $1, \cdots, m$, and $\xi / \rho_{k}$ has at most one member $\xi^{\prime}$. Also the proof of Lemma 3 shows that each $\eta_{i} / \rho_{k}$ has at most one member $\eta_{i}^{\prime}\left(\right.$ for $i=1, \cdots, m$ ) since $\rho_{k}$ is part of an MCD.

(i) If $\left\{\eta_{1}^{\prime}, \cdots, \eta_{m}^{\prime}\right\}$ is empty, that is $\rho_{k}$ is a complete reduction of $\left\{\eta_{1}, \cdots, \eta_{m}\right\}$, end the sequence at $\rho_{k}$.

(ii) If $\left\{\eta_{1}^{\prime}, \cdots, \eta_{m}^{\prime}\right\}$ is not empty but there are no $\eta_{i}^{\prime} \prec \xi^{\prime}$, choose any cell $\zeta$ minimal in $\left\{\eta_{1}^{\prime}, \cdots, \eta_{m}^{\prime}\right\}$ and define $\rho_{k+1}=\rho_{k}+\zeta$.

(iii) If there are some $\eta_{i}^{\prime} \prec \xi^{\prime}$, choose $\zeta$ to be the one of these $\eta_{i}^{\prime}$ given by (A6) and define $\rho_{k+1}=\rho_{k}+\zeta$. $\zeta$ is minimal in $\left\{\eta_{1}^{\prime}, \cdots, \eta_{m}^{\prime}\right\}$ because for those $\eta_{t}^{\prime} \prec \xi^{\prime}$, (A6) implies that $\eta_{i}^{\prime} K \zeta$, and for those $\eta_{i}^{\prime} K \xi^{\prime}$, (A2) and the fact that $\zeta \prec \xi^{\prime}$ imply that $\eta_{i}^{\prime} K \zeta$.

\footnotetext{
${ }^{4}$ In terms of $\lambda$-reduction, a $\xi$-MCD is an MCD in which contractions in $\xi$ are done first (in a certain order), then contractions which do not overlap $\xi$, and finally contractions of parts containing $\xi$.
} 
For the second part, $\sigma$, of the $\xi$-MCD, notice that by Lemma $5, \eta_{m+s} / \rho$ has at most one member $\eta_{m+1}^{\prime}$, for $j=1, \cdots,(n-m)$. Also by Lemma 5 ,

$$
h>j \Rightarrow \eta_{m+h} / \rho \nless \eta_{m+j} / \rho
$$

since $\rho$ is a development (perhaps not complete) of $\left\{\eta_{1}, \cdots, \eta_{m}, \eta_{m+j}\right\}$ and $\eta_{m+h} K \eta_{1}$ for $i=1, \cdots, m$ and for $i=m+j$. In other words,

$$
h>j \Rightarrow \eta_{m+h}^{\prime} K \eta_{m+1}^{\prime}
$$

80 the reduction of $\left\{\eta_{m+1}^{\prime}, \cdots, \eta_{n}^{\prime}\right\}$ defined as in Lemma 7 will be an MCD. Choose $\sigma$ to be this reduction, but if $m=n$, choose $\sigma$ to be 0 .

It can be seen from the definition that any set $\left\{\eta_{1}, \cdots, \eta_{n}\right\}$ will have at least one $\xi$-MCD for each $\xi$ coinitial with the set.

With the tools now built up, the main part of the proof of the theorem can begin. By Lemma 1 it is enough to prove $\left(C_{1}\right)$, which says that if a cell $\xi$ and an MCD $\rho$ are coinitial, then there exist MCDs $\tau$ and $\sigma$ such that $\rho+\sigma \simeq \xi+\tau$. Let $\left\{\eta_{1}, \cdots, \eta_{n}\right\}$ be a finite set of cells of which $\rho$ is an MCD, and let $\rho^{*}$ be a $\xi$-MCD of $\left\{\eta_{1}, \cdots, \eta_{n}\right\}$. By Lemma $8, \rho^{*} \simeq \rho$, so, replacing $\rho$ by $\rho^{*}$ in $\left(C_{1}\right)$, it is enough to prove

$\left(C_{2}\right)$. If $\xi, \eta_{1}, \cdots, \eta_{n}$ are mutually coinitial and $\rho$ is a $\xi-M C D$ of $\left\{\eta_{1}, \cdots, \eta_{n}\right\}$, then there exist an $M C D, \tau$, of $\left\{\eta_{1} / \xi, \cdots, \eta_{n} / \xi\right\}$ and an $M C D, \sigma$, such that $\rho+\sigma \simeq$ $\xi+\tau$.

The proof of this property will be split into several parts, according to the relationships between $\eta_{1}, \cdots, \eta_{n}$ and $\xi$.

LEMMA 9. $\left(\mathrm{C}_{2}\right)$ is true if either

(i) for $i=1, \cdots, n, \xi K \eta_{i}$ and $\xi \neq \eta_{1}$ or

(ii) for some $i, \xi=\eta_{i}$.

Furthermore, in these two cases $\sigma$ is an $M C D$ of the set $\xi / \rho$, and if $\zeta$ is any cell coinitial with $\xi$ such that $\zeta K \xi$ and $\zeta K \eta_{i}$ for $i=1, \cdots, n$, then $\zeta / \rho+\sigma=\zeta / \xi+\tau$.

The proof is by induction on $n$; the clause about $\zeta$ has been put in just to make the induction-step work.

Basis. When $n=0$ and so $\rho=0$ : choose $\tau=0$ and $\sigma=\xi$. Then $\tau$ is an MCD of the empty set and $\sigma$ is an MCD of the set $\{\xi\}$ which is the same as $\xi / \rho$.

Case (i) of the induction-step. Assume that $n>0$ and that the lemma is true in both cases (i) and (ii) for all $n^{\prime} \leq n-1$; suppose that $\xi, \eta_{1}, \cdots, \eta_{n}, \rho$ and $\zeta$ satisfy the assumptions in the lemma, and that $\xi K \eta_{1}$ and $\xi \neq \eta_{1}$ for $i=1, \cdots, n$. Renumber the $\eta_{1}, \cdots, \eta_{n}$ so that the first cell of $\rho$ is $\eta_{1}$; this cell will be called $\eta$

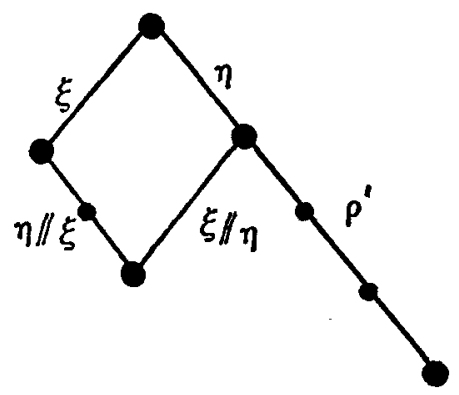

FIGURe 6 
for short. Then $\rho=\eta+\rho^{\prime}$ for some reduction $\rho^{\prime}$. By (A3), $\xi / \eta$ has at most one member, and by (A7), $\xi+\eta / / \xi \simeq \eta+\xi / / \eta$. (See Figure 6.)

Also

(1)

$$
\eta_{i} / \xi K \eta / \xi \text { for } i=2, \cdots, n \text {. }
$$

Proof. Since $\rho$ is an MCD, $\eta_{i} K \eta$ for $i=2, \cdots, n$.

If $\eta_{i} K \xi$, then by (A5), $\eta_{i} / \xi K \eta / \xi$.

If $\eta_{1} \prec \xi$, then by the assumption that $\rho$ is a $\xi$-MCD, $\eta$ must have been chosen by clause (iii) of Definition 1 . Hence $\eta_{i} / \xi K \eta / \xi$ by (A6).

$$
\eta_{t} /(\xi+\eta / / \xi)=\eta_{i} /(\eta+\xi / / \eta) \text { for } i=2, \cdots, n \text {. }
$$

Proor. As for (1), $\eta_{i} K \eta$ for $i=2, \cdots, n$.

If $\eta_{i} K \xi$, then use (A8) part (i).

If $\eta_{i} \prec \xi$, then as for (1), $\eta$ must have been chosen by Definition 1(iii), and hence $\eta \prec \xi$. Now use part (ii) of (A8), together with (1).

Further, by part (i) of (A8)

$$
\zeta /(\xi+\eta / / \xi)=\zeta /(\eta+\xi / / \eta) \text {. }
$$

Now by (A3) there are no more than $n-1$ members of $\left\{\eta_{2} / \eta, \cdots, \eta_{n} / \eta\right\}$. If any of $\xi / \eta, \zeta / \eta, \eta_{2} / \eta, \cdots, \eta_{n} / \eta$ is not empty, suppose its sole member is $\xi^{\prime}, \zeta^{\prime}, \eta_{2}^{\prime}, \cdots$, or $\eta_{n}^{\prime}$ respectively. By (A5) applied three times; for $i=2, \cdots, n$,

$$
\zeta / \eta \nless \eta_{1} / \eta, \quad \xi / \eta K \eta_{1} / \eta, \quad \zeta / \eta \nless \xi / \eta \text {. }
$$

The proof now splits up into two subcases, according as $\xi / \eta$ is empty or not.

Subcase (I) (see Figure 7). When $\xi / \eta$ is not empty, then its sole member is $\xi^{\prime}$. By Definition 1 there exists a $\xi^{\prime}-\mathrm{MCD}, \rho^{\prime \prime}$, of $\left\{\eta_{2}^{\prime}, \cdots, \eta_{n}^{\prime}\right\}$. By Lemma 8 and (4),

$$
\rho^{\prime} \simeq \rho^{\prime \prime}, \quad \xi^{\prime} / \rho^{\prime}=\xi^{\prime} / \rho^{\prime \prime}, \quad(\zeta / \eta) / \rho^{\prime}=(\zeta / \eta) / \rho^{\prime \prime} .
$$

By (4), the induction-hypothesis can be applied to $\xi^{\prime}, \zeta^{\prime},\left\{\eta_{2}^{\prime}, \cdots, \eta_{n}^{\prime}\right\}$ and $\rho^{\prime \prime}$. (Case (ii) of the induction-hypothesis is applied if $\xi^{\prime}=\eta_{i}^{\prime}$ for some $i$, otherwise Case (i) is used.) Hence there exist an MCD, $\tau^{\prime}$, of $\left\{\eta_{2}^{\prime} / \xi^{\prime}, \cdots, \eta_{n}^{\prime} / \xi^{\prime}\right\}$ and an MCD, $\sigma$, of $\xi^{\prime} / \rho^{\prime \prime}$ such that $\rho^{\prime \prime}+\sigma \simeq \xi^{\prime}+\tau^{\prime}$ and $\zeta^{\prime} /\left(\rho^{\prime \prime}+\sigma\right)=\zeta^{\prime} /\left(\xi^{\prime}+\tau^{\prime}\right)$.

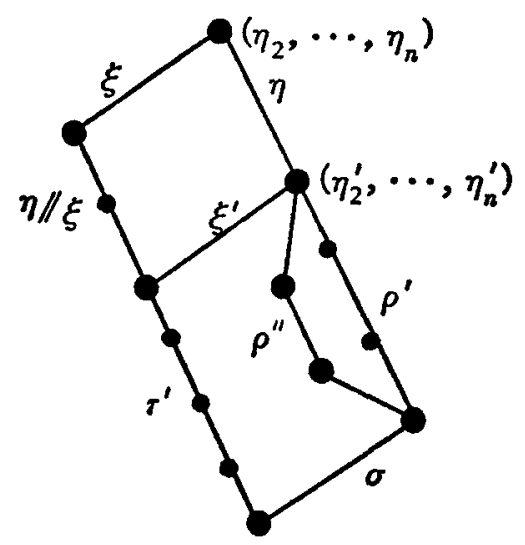

FIOURE 7 
Define $\tau$ to be $(\eta / / \xi)+\tau^{\prime}$. Hence

$$
\xi+\tau=\xi+(\eta / / \xi)+\tau^{\prime} \simeq \eta+\xi^{\prime}+\tau^{\prime} \simeq \eta+\rho^{\prime \prime}+\sigma \simeq \eta+\rho^{\prime}+\sigma=\rho+\sigma .
$$

Now by (2), if $\eta_{i} / \eta$ is not empty,

$$
\eta_{i}^{\prime} / \xi^{\prime}=\left(\eta_{i} / \eta\right) /(\xi / \eta)=\eta_{1} /(\eta+\xi / \eta)=\eta_{i} /(\xi+\eta / / \xi)
$$

so $\tau^{\prime}$ is an MCD of $\left\{\eta_{2} /(\xi+\eta / / \xi), \cdots, \eta_{n} /(\xi+\eta / / \xi)\right\}$; hence by (1) and Lemma 6, $\tau$ is an MCD of $\left\{\eta_{1} / \xi, \cdots, \eta_{n} / \xi\right\}$, as required. Also

$$
\xi^{\prime} / \rho^{\prime \prime}=\xi^{\prime} / \rho^{\prime}=(\xi / \eta) / \rho^{\prime}=\xi /\left(\eta+\rho^{\prime}\right)=\xi / \rho
$$

so $\sigma$ is an $\mathrm{MCD}$ of $\xi / \rho$.

For the derivatives of $\zeta:$ if $\zeta / \eta$ is not empty, then

$$
\begin{aligned}
\zeta /(\rho+\sigma) & =\zeta /\left(\eta+\rho^{\prime}+\sigma\right)=\zeta^{\prime} /\left(\rho^{\prime}+\sigma\right)=\zeta^{\prime} /\left(\rho^{\prime \prime}+\sigma\right) \\
& =\zeta^{\prime} /\left(\xi^{\prime}+\tau^{\prime}\right) \text { by the induction-hypothesis applied to } \rho^{\prime \prime} \\
& =(\zeta /(\eta+\xi / \eta)) / \tau^{\prime}=(\zeta /(\xi+\eta / / \xi)) / \tau^{\prime} \quad \text { by }(3) \\
& =\zeta /(\xi+\tau) \text { as required. }
\end{aligned}
$$

If $\zeta / \eta$ is empty, then $\zeta /(\eta+\xi / / \eta)$ will be empty, and by (2), so will $\zeta /(\xi+\eta / / \xi)$. Therefore $\zeta /(\xi+\tau)$ will be empty; $\zeta /(\rho+\sigma)$ will also be empty because it is the same as $(\zeta / \eta) /\left(\rho^{\prime}+\sigma\right)$.

Subcase (II) (see Figure 8 ). When $\xi / \eta$ is empty, then so is $\xi / \rho$. Therefore $\eta=\eta+0=\eta+\xi / / \eta \simeq \xi+\eta / / \xi$ and by $(2), \eta_{i} /(\xi+\eta / / \xi)=\eta_{i} /(\eta+\xi / / \eta)=\eta_{i} / \eta$ for $i=2, \cdots, n$.

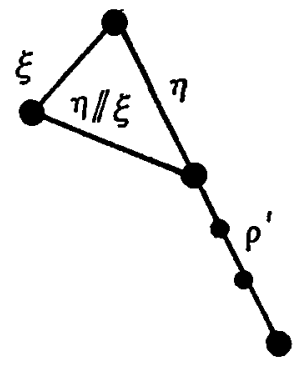

Figure 8

Choose $r$ to be $(\eta / / \xi)+\rho^{\prime}$ and $\sigma$ to be 0 . The rest of the reasoning is the same as in Subcase (I), replacing $\xi^{\prime}$ by 0 and $\tau^{\prime}$ by $\rho^{\prime}$, and letting $\rho^{\prime \prime}=\rho^{\prime}$.

Case (ii) of the induction-step. Assuming the lemma, in both cases, for all $n^{\prime} \leq n-1$, suppose that $\xi, \eta_{1}, \cdots, \eta_{n}, \rho$ and $\zeta$ satisfy the assumptions in the lemma, and that $\xi=\eta_{i}$ for some $i$. Then $\xi=\eta_{m+1}$, where $m$ is defined in the construction of $\rho$ by Definition 1 . By this construction, there exist a $\xi-\mathrm{MCD}, \rho^{\prime}$, of $\left\{\eta_{1}, \cdots, \eta_{m}\right\}$ and an MCD, $\rho "$, such that

if $\xi / \rho^{\prime}$ contains only one cell $\xi^{\prime}$, then $\rho=\rho^{\prime}+\xi^{\prime}+\rho^{\prime \prime}$ and

if $\xi / \rho^{\prime}$ is empty, then $\rho=\rho^{\prime}+0+\rho^{\prime \prime}$. (See Figure 9.)

Now $m \leq n-1$, so applying Case (i) of the induction-hypothesis to $\left\{\eta_{1}, \cdots, \eta_{m}\right\}$, $\xi$ and $\rho^{\prime}$, gives an MCD $\tau^{\prime}$ of $\left\{\eta_{1} / \xi, \cdots, \eta_{m} / \xi\right\}$ and an MCD $\sigma^{\prime}$ of $\xi / \rho^{\prime}$ such that

$$
\xi+\tau^{\prime} \simeq \rho^{\prime}+\sigma^{\prime} \text { and } \zeta^{*} /\left(\xi+\tau^{\prime}\right)=\zeta^{*} /\left(\rho^{\prime}+\tau^{\prime}\right)
$$

for every cell $\zeta^{*}$ such that $\zeta^{*} K \eta_{i}$ for $i=1, \cdots, m$ and $\zeta^{*} K \xi$. 


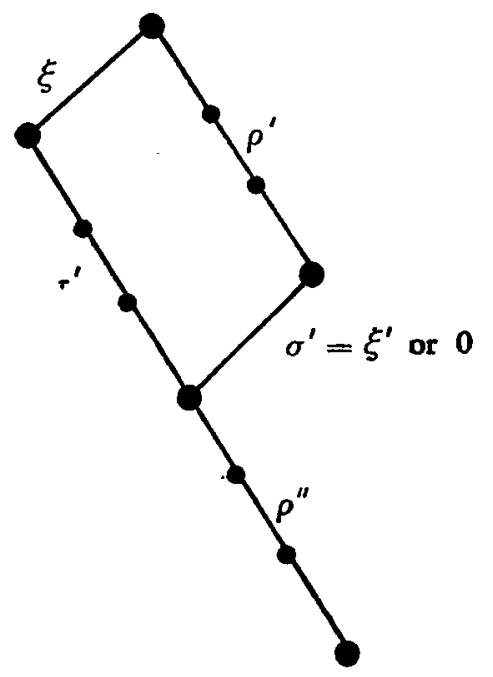

Figure 9

Since $\xi / \rho^{\prime}$ has at most one member, $\xi^{\prime}$, the MCD $\sigma^{\prime}$ must be either 0 or $\xi^{\prime}$ according as $\xi / \rho^{\prime}$ is empty or not. Therefore by a previous remark, $\rho=\rho^{\prime}+\sigma^{\prime}+\rho^{\prime \prime}$. Also, by its definition, $\rho^{\prime \prime}$ is an MCD of $\left\{\eta_{m+2} /\left(\rho^{\prime}+\sigma^{\prime}\right), \cdots, \eta_{n} /\left(\rho^{\prime}+\sigma^{\prime}\right)\right\}$. Choose $\tau$ to be $\left(\tau^{\prime}+\rho^{\prime \prime}\right)$ and $\sigma$ to be 0 . Then

$$
\xi+\tau=\xi+\tau^{\prime}+\rho^{\prime \prime} \simeq \rho^{\prime}+\sigma^{\prime}+\rho^{\prime \prime}=\rho=\rho+0=\rho+\sigma .
$$

Also $\sigma$ is an MCD of $\xi / \rho$, because $\xi / \rho=\eta_{m+1} / \rho$ which is empty, since $\rho$ is a complete development.

The reduction $\tau$ is an MCD of $\left\{\eta_{1} / \xi, \cdots, \eta_{n} / \xi\right\}$.

Proof. The second part of $\tau$ is $\rho^{\prime \prime}$, which is an MCD of

$$
\left\{\eta_{m+2} /\left(\rho^{\prime}+\sigma^{\prime}\right), \cdots, \eta_{n} /\left(\rho^{\prime}+\sigma^{\prime}\right)\right\} \text {. }
$$

Now for $j=2, \cdots,(n-m)$,

$$
\begin{aligned}
\eta_{m+j} /\left(\rho^{\prime}+\sigma^{\prime}\right) & =\eta_{m+j} /\left(\xi+\tau^{\prime}\right) \text { by (5) with } \eta_{m+j} \text { as } \zeta^{*}, \\
& =\left(\eta_{m+j} / \xi\right) / \tau^{\prime} .
\end{aligned}
$$

Also $\eta_{m+j} / \xi K \eta_{i} / \xi$ for $i=1, \cdots, m$ by (A5). Hence by Lemma $6, \tau$ is an MCD of $\left\{\eta_{1} / \xi, \cdots, \eta_{m} / \xi, \eta_{m+2} / \xi, \cdots, \eta_{n} / \xi\right\}$, which is the same as $\left\{\eta_{1} / \xi, \cdots, \eta_{n} / \xi\right\}$ by (A4), since $\eta_{m+1}=\xi$.

As for the derivatives of $\zeta$;

$$
\begin{aligned}
\zeta /(\xi+\tau) & =\zeta /\left(\xi+\tau^{\prime}+\rho^{\prime \prime}\right)=\zeta /\left(\rho^{\prime}+\sigma^{\prime}+\rho^{\prime \prime}\right) \quad \text { by }(5) \text { with } \zeta \text { as the } \zeta^{*}, \\
& =\zeta / \rho=\zeta /(\rho+\sigma),
\end{aligned}
$$

as required.

LEMmA 10. $\left(\mathrm{C}_{2}\right)$ is true if $\eta_{1} K \xi$ and $\eta_{i} \neq \xi$ for $i=1, \cdots, n$. Further, $\sigma$ is an $M C D$ of a subset of $\xi / \rho$, and if $\zeta$ is any cell coinitial with $\xi$ such that $\zeta K \xi$ and $\zeta K \eta_{i}$ for $i=1, \cdots, n$, then $\zeta /(p+\sigma)=\zeta /(\xi+\tau)$.

The proof is by induction on $n$; notice that $\sigma$ might not be an MCD of the whole of $\xi / \rho .^{5}$

But if all derivative sets are finite, $\sigma$ can be made an MCD of the whole set. 
BASIS. When $n=0$ : let $\tau=0$ and $\sigma=\xi$.

Induction-step. When $n>0$ : if there are no $\eta_{t}$ with $\xi<\eta_{i}$, use Case (i) of Lemma 9. Otherwise, using Definition $1, \rho=\rho^{\prime}+\rho^{*}$, where $\rho^{\prime}$ is a $\xi$-MCD of $\left\{\eta_{1}, \cdots, \eta_{n-1}\right\}$, and $\rho^{*}$ is either 0 or $\eta^{\prime}$ according as $\eta_{n} / \rho^{\prime}$ is empty or has one member $\eta^{\prime}$. Also $\xi \prec \eta_{n}$ and $\eta_{n} K \eta_{i}$ for $i=1, \cdots,(n-1)$, by Definition 1 . Call $\eta_{n}$ " $\eta$ " for short.

By the induction-hypothesis applied to $\xi, \rho^{\prime}$ and $\left\{\eta_{1}, \cdots, \eta_{n-1}\right\}$, there exist an MCD $\tau^{\prime}$ of $\left\{\eta_{1} / \xi, \cdots, \eta_{n-1} / \xi\right\}$, and an MCD $\sigma^{\prime}$ of a subset of $\xi / \rho^{\prime}$ such that

$$
\xi+\tau^{\prime} \simeq \rho^{\prime}+\sigma^{\prime} \text { and } \zeta^{*} /\left(\xi+\tau^{\prime}\right)=\zeta^{*} /\left(\rho^{\prime}+\sigma^{\prime}\right)
$$

for any cell $\zeta^{*}$ such that $\zeta^{*} K \xi$ and $\zeta^{*} K \eta_{1}$ for $i=1, \cdots, n-1$. (See Figure 10.)

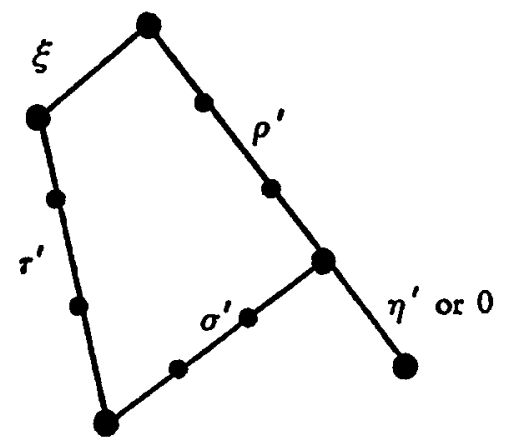

FIGURE 10

Now $\eta K \xi$ since $\xi \prec \eta$, so by (6) applied to $\eta$;

$$
\eta /\left(\xi+\tau^{\prime}\right)=\eta /\left(\rho^{\prime}+\sigma^{\prime}\right) .
$$

If $\eta / \rho^{\prime}$ is empty, then $\rho=\rho^{\prime}$; in this case define $\sigma=\sigma^{\prime}$ and $\tau=\tau^{\prime}$. Hence $\xi+\tau \simeq$ $\rho+\sigma$ and $\zeta /(\xi+\tau)=\zeta /(\rho+\sigma)$ by (6). Also $\sigma$ is an MCD of a subset of $\xi / \rho^{\prime}$, which is the same as $\xi / \rho$. Finally, $\tau$ would be an MCD of $\left\{\eta_{1} / \xi, \cdots, \eta_{n} / \xi\right\}$ if $\left(\eta_{n} / \xi\right) / \tau$ were empty and at each stage $\tau^{*}$ of $\tau,\left(\eta_{n} / \xi\right) / \tau^{*} K\left(\eta_{l} / \xi\right) / \tau^{*}$. (A stage of $\tau$ is any reduction $\tau^{*}$ such that $\tau=\tau^{*}+\tau_{1}$ for some $\tau_{1}$.) The former is true because $\eta /\left(\rho^{\prime}+\sigma^{\prime}\right)$ is empty and by $(7)$ is the same as $\eta /(\xi+\tau)$, which is $\left(\eta_{n} / \xi\right) / \tau$. The latter is true by Lemma 5 , since $\eta_{n} / \xi K \eta_{1} / \xi$ by (A5).

From now on, assume that $\eta / \rho^{\prime}$ is not empty. Then

$$
\eta / \rho^{\prime} K \xi / \rho^{\prime} \text { and } \zeta / \rho^{\prime} K \eta / \rho^{\prime} \text { and } \zeta / \rho^{\prime} K \xi / \rho^{\prime} \text {. }
$$

PRoof. $\rho^{\prime}$ is an MCD of $\left\{\eta_{1}, \cdots, \eta_{n-1}\right\}$ and hence is a development of $\left\{\xi, \eta_{1}, \cdots, \eta_{n-1}\right\}$. Since $\eta \nless \xi$ and $\eta \nless \eta_{i}$ for $i=1, \cdots, n-1$, Lemma 5 implies that $\eta / \rho^{\prime} K \xi / \rho^{\prime}$. Similarly $\zeta / \rho^{\prime} K \xi / \rho^{\prime} . \rho^{\prime}$ is also a development of $\left\{\eta, \eta_{1}, \cdots, \eta_{n-1}\right\}$ so by Lemma $5, \zeta / \rho^{\prime} K \eta / \rho^{\prime}$.

Let $\left\{\xi_{1}, \cdots, \xi_{h}\right\}(h \geq 0)$ be the members of $\xi / \rho^{\prime}$ whose derivatives are the cells of $\sigma^{\prime}$ (Lemma 4 ensures that such a finite set does exist); then $\sigma^{\prime}$ is an MCD of $\left\{\xi_{1}, \cdots, \xi_{h}\right\}$. Also let $\eta^{\prime}$ be the sole member of $\eta / \rho^{\prime}$, and $\zeta^{\prime}$ be the sole member of $\zeta / \rho^{\prime}$ if that set is not empty. By (8), $\eta^{\prime} K \xi_{i}$ and $\zeta^{\prime} K \xi_{i}$ for $i=1, \cdots, h$, and $\zeta^{\prime} K \eta^{\prime}$ (if $\zeta^{\prime}$ exists). 
Now by Definition 1 there exists an $\eta^{\prime}$-MCD, $\sigma^{*}$, of $\left\{\xi_{1}, \cdots, \xi_{n}\right\}$, and by Lemma $8, \sigma^{\prime} \simeq \sigma^{*}$ and $\eta^{\prime} / \sigma^{*}=\eta^{\prime} / \sigma^{\prime}$, because $\eta^{\prime} K \xi_{l}$ for $i=1, \cdots, h$. Similarly $\zeta^{\prime} / \sigma^{*}=$ $\zeta^{\prime} / \sigma^{\prime}$. (See Figure 11.)

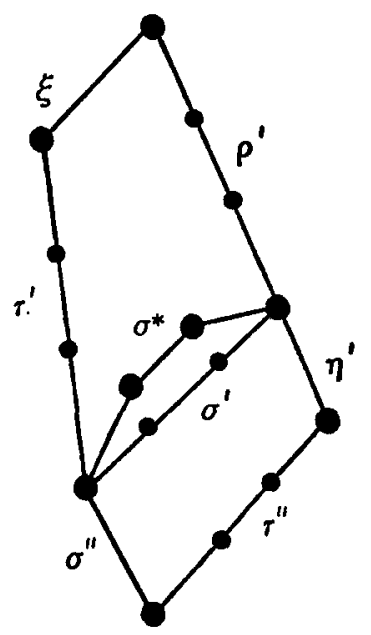

Figure 11

Lemma 9 can be applied to $\zeta^{\prime}, \eta^{\prime},\left\{\xi_{1}, \cdots, \xi_{h}\right\}$ and $\sigma^{*}$ (using Case (ii) or (i) according as $\eta^{\prime}$ is or is not one of $\left.\xi_{1}, \cdots, \xi_{h}\right)$ to obtain an MCD, $\tau^{\prime \prime}$, of $\left\{\xi_{1} / \eta^{\prime}, \cdots, \xi_{n} / \eta^{\prime}\right\}$ and an MCD, $\sigma^{\prime \prime}$, of $\eta^{\prime} / \sigma^{*}$ such that

$$
\sigma^{*}+\sigma^{\prime \prime} \simeq \eta^{\prime}+\tau^{\prime \prime} \text { and } \zeta^{\prime} /\left(\sigma^{*}+\sigma^{\prime \prime}\right)=\zeta^{\prime} /\left(\eta^{\prime}+\tau^{\prime \prime}\right) \text { if } \zeta^{\prime} \text { exists. }
$$

Define $\tau$ to be $\tau^{\prime}+\sigma^{\prime \prime}$ and $\sigma$ to be $\tau^{\prime \prime}$. Then $\sigma$ is an MCD of $\left\{\xi_{1} / \eta^{\prime}, \cdots, \xi_{n} / \eta^{\prime}\right\}$, which is a subset of $\left(\xi / \rho^{\prime}\right) / \eta^{\prime}$, which is $\xi / \rho$. By Lemma $6, \tau$ will be an MCD of $\left\{\eta_{1} / \xi, \cdots, \eta_{n} / \xi\right\}$ if $\eta_{n} / \xi K \eta_{i} / \xi$ for $i=1, \cdots, n-1$ and $\sigma^{\prime \prime}$ is an MCD of $\left(\eta_{n} / \xi\right) / \tau^{\prime}$. But the former is true by (A5), and for the latter, $\sigma^{\prime \prime}$ is an MCD of $\eta^{\prime} / \sigma^{*}$ which is the same as $\eta /\left(\xi+\tau^{\prime}\right)$ because $\eta^{\prime} / \sigma^{*}=\eta^{\prime} / \sigma^{\prime}=\left(\eta / \rho^{\prime}\right) / \sigma^{\prime}=\eta /\left(\rho^{\prime}+\sigma^{\prime}\right)=\eta /\left(\xi+\tau^{\prime}\right)$ by (7). So $\tau$ is an MCD of $\left\{\eta_{1} / \xi, \cdots, \eta_{n} / \xi\right\}$. Also

$$
\begin{aligned}
\xi+\tau & =\left(\xi+\tau^{\prime}+\sigma^{\prime \prime}\right) \simeq\left(\rho^{\prime}+\sigma^{\prime}+\sigma^{\prime \prime}\right) \\
& \simeq\left(\rho^{\prime}+\sigma^{*}+\sigma^{\prime \prime}\right) \simeq\left(\rho^{\prime}+\eta^{\prime}+\tau^{\prime \prime}\right)=\rho+\sigma .
\end{aligned}
$$

It remains to show that the derivatives of $\zeta$ are the same with respect to $\xi+\tau$ and $\rho+\sigma$. If $\zeta / \rho^{\prime}$ is empty, then so will be $\zeta /(\rho+\sigma)$. Also $\zeta /\left(\rho^{\prime}+\sigma^{\prime}\right)$ and hence by (6), $\zeta /\left(\xi+\tau^{\prime}\right)$, will be empty. Therefore $\zeta /(\xi+\tau)$ will be empty too. Finally, if $\zeta / \rho^{\prime}$ is not empty, and $\zeta^{\prime}$ is its sole member, then

$$
\begin{aligned}
\zeta /(\rho+\sigma) & =\zeta /\left(\rho^{\prime}+\eta^{\prime}+\sigma\right)=\zeta^{\prime} /\left(\eta^{\prime}+\sigma\right)=\zeta^{\prime} /\left(\sigma^{*}+\sigma^{\prime \prime}\right) \text { by }(9), \text { since } \sigma=\tau^{\prime \prime} \\
& =\left(\zeta^{\prime} / \sigma^{*}\right) / \sigma^{\prime \prime}=\left(\zeta^{\prime} / \sigma^{\prime}\right) / \sigma^{\prime \prime}=\zeta^{\prime} /\left(\sigma^{\prime}+\sigma^{\prime \prime}\right)=\zeta /\left(\rho^{\prime}+\sigma^{\prime}+\sigma^{\prime \prime}\right) \\
& =\zeta /\left(\xi+\tau^{\prime}+\sigma^{\prime \prime}\right) \text { by }(6), \\
& =\zeta /(\xi+\tau),
\end{aligned}
$$

completing the proof of Lemma 10.

LEMMA 11. $\left(\mathrm{C}_{2}\right)$ is true if $\xi \neq \eta_{1}$ for $i=1, \cdots, n$.

Proof. Suppose that $\xi,\left\{\eta_{1}, \cdots, \eta_{n}\right\}$ and $\rho$ are as in $\left(C_{2}\right)$. Then since $\rho$ is a 
$\xi$-MCD, Definition 1 gives a number $m$, a $\xi$-MCD $\rho^{\prime}$ of $\left\{\eta_{1}, \cdots, \eta_{m}\right\}$, and an MCD $\rho^{\prime \prime}$ of $\left\{\eta_{m+1} / \rho^{\prime}, \cdots, \eta_{n} / \rho^{\prime}\right\}$ such that $\rho=\rho^{\prime}+\rho^{\prime \prime}$. Also, by definition of $m, \xi K \eta_{1}$ and $\eta_{m+1} K \eta_{1}$ for $i=1, \cdots, m$ and $j=1, \cdots,(n-m)$, so there must be at most one member in each of $\xi / \rho^{\prime}$ and $\eta_{m+j} / \rho^{\prime}$, by Lemma 5 . Further, by a proof like that of (8), with $\eta_{m+j}$ instead of $\eta$;

$$
\eta_{m+s} / \rho^{\prime} K \xi / \rho^{\prime}
$$

(Here, $\eta_{m+j} K \xi$ because $\xi \prec \eta_{m+s}$ by definition of $m$.)

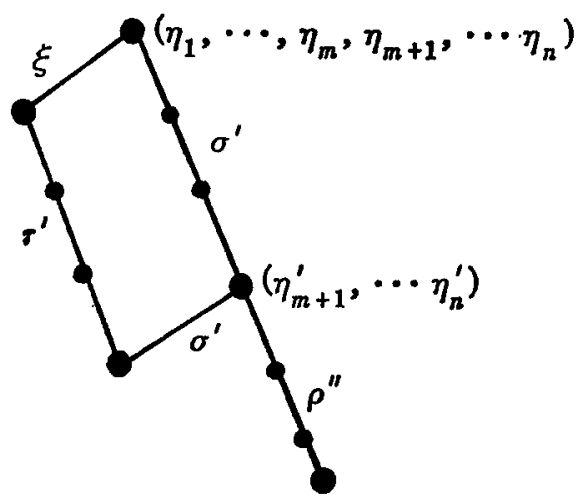

Figure 12

Lemma 9(i) applied to $\xi, \eta_{1}, \cdots, \eta_{m}$ and $\rho^{\prime}$ gives an MCD, $\tau^{\prime}$, of $\left\{\eta_{1} / \xi, \cdots, \eta_{m} / \xi\right\}$ and an $\mathrm{MCD}, \sigma^{\prime}$, of $\xi / \rho^{\prime}$ such that

$$
\rho^{\prime}+\sigma^{\prime} \simeq \xi+\tau^{\prime} \text { and } \zeta^{*} /\left(\rho^{\prime}+\sigma^{\prime}\right)=\zeta^{*} /\left(\xi+\tau^{\prime}\right)
$$

for any cell $\zeta^{*}$ such that $\zeta^{*} K \xi$ and $\zeta^{*} K \eta_{i}$ for $i=1, \cdots, m$. (See Figure 12.) Now $\eta_{m+j}$ satisfies the conditions of (11), so

$$
\eta_{m+j} /\left(\rho^{\prime}+\sigma^{\prime}\right)=\eta_{m+j} /\left(\xi+\tau^{\prime}\right) \text {. }
$$

Define $\eta_{m+1}^{\prime}, \cdots, \eta_{n}^{\prime}, \xi^{\prime}$ to be the sole members of $\eta_{m+1} / \rho^{\prime}, \cdots, \eta_{n} / \rho^{\prime}, \xi / \rho^{\prime}$ respectively, for each of these sets which is not empty. Since $\sigma^{\prime}$ is an MCD of $\xi / \rho^{\prime}, \sigma^{\prime}$ must be 0 or $\xi^{\prime}$ according as $\xi / \rho^{\prime}$ is or is not empty.

Case 1. Suppose that $\xi / \rho^{\prime}$ is not empty. Then by Definition 1 there exists a $\xi^{\prime}-\mathrm{MCD}, \rho^{*}$, of $\left\{\eta_{m+1}^{\prime}, \cdots, \eta_{n}^{\prime}\right\}$ and by Lemma $8, \rho^{*} \simeq \rho^{\prime \prime}$. By $(10), \eta_{m+1}^{\prime} K \xi^{\prime}$ for $j=1, \cdots,(n-m)$. Therefore, Lemma 10 (or Lemma 9(ii) if $\xi^{\prime}$ is one of $\left.\eta_{m+1}^{\prime}, \cdots, \eta_{n}^{\prime}\right)$ can be applied to $\xi^{\prime}, \eta_{m+1}^{\prime}, \cdots, \eta_{n}^{\prime}$ and $\rho^{*}$ to give an MCD, $\tau^{\prime \prime}$, of $\left\{\eta_{m+1}^{\prime} / \xi^{\prime}, \cdots, \eta_{n}^{\prime} / \xi^{\prime}\right\}$ and an MCD, $\sigma$, of a subset of $\xi^{\prime} / \rho^{*}$ such that $\xi^{\prime}+\tau^{\prime \prime} \simeq$ $\rho^{*}+\sigma$. (See Figure 13.)

Define $\tau$ to be $\tau^{\prime}+\tau^{\prime \prime}$. Now $\tau^{\prime \prime}$ is an MCD of $\left\{\left(\eta_{m+1} / \xi\right) / \tau^{\prime}, \cdots,\left(\eta_{n} / \xi\right) / \tau^{\prime}\right\}$ because for $j=1, \cdots, n-m$,

$$
\begin{aligned}
\eta_{m+j}^{\prime} / \xi^{\prime} & =\eta_{m+j} /\left(\rho^{\prime}+\xi^{\prime}\right)=\eta_{m+j} /\left(\xi+\tau^{\prime}\right) \quad \text { by }(12), \text { since } \sigma^{\prime}=\xi^{\prime} ; \\
& =\left(\eta_{m+j} / \xi\right) / \tau^{\prime} .
\end{aligned}
$$






FigurR 13

Also for $i=1, \cdots, m$ and $j=1, \cdots, n-m, \eta_{m+j} / \xi K \eta_{l} / \xi$ by (A5), so by Lemma $6, \tau$ is an MCD of $\left\{\eta_{1} / \xi, \cdots, \eta_{n} / \xi\right\}$. Finally,

$\xi+\tau=\left(\xi+\tau^{\prime}+\tau^{\prime \prime}\right) \simeq\left(\rho^{\prime}+\sigma^{\prime}+\tau^{\prime \prime}\right)$

$$
\simeq\left(\rho^{\prime}+\xi^{\prime}+\tau^{\prime \prime}\right) \simeq\left(\rho^{\prime}+\rho^{*}+\sigma\right) \simeq\left(\rho^{\prime}+\rho^{\prime \prime}+\sigma\right)=\rho+\sigma .
$$

Case 2. Suppose that $\xi / \rho^{\prime}$ is empty. In this case, $\sigma^{\prime}=0$; define $\sigma$ to be 0 and $\tau$ to be $r^{\prime}+\rho^{\prime \prime}$. (See Figure 14.)



FIGURe 14

The rest of the reasoning is the same as in Case 1 , replacing $\xi^{\prime}$ by 0 and $\tau^{\prime \prime}$ by $\rho^{\prime \prime}$, and letting $\rho^{*}$ be $\rho^{\prime \prime}$.

Now Lemmas 11 and 9(ii) together imply that $\left(C_{2}\right)$ is true in all possible cases, so the proof of Theorem 1 is complete.

\$4. Relation of Theorem 1 to Chapter 4 of [3]. In [3, Chapter 4], Curry deduced the Church-Rosser property from certain properties $\left(H_{0}\right), \cdots,\left(H_{7}\right)$. By interpreting the relation " $\prec$ " as Curry's relation "f" it can be shown that any system 
satisfying $\left(H_{0}\right), \cdots,\left(H_{7}\right)$ also satisfies (A1), $\cdots,(\mathrm{A} 8)$. Also a system which satisfies (A1), $\cdots$, (A8) but not $\left(H_{0}\right), \cdots,\left(H_{7}\right)$ can be constructed, using the fact that $\left(\mathrm{H}_{2}\right)$ is more restrictive than the corresponding assumption (A3). However, Curry actually deduced more than the Church-Rosser property from his assumptions: he showed that any two developments of a finite coinitial set of cells have the same terminus. I have not been able to deduce this from (A1), , , (A8).

\section{REFERENCES}

[1] H. B. CURRY, A new proof of the Church-Rosser Theorem, Koninklijke Nederlandse Akademie van Wetenschappen. Proceedings. Series A, vol. 55 (1952), pp. 16-22.

[2] A. Church, The calculi of Lambda-conversion, Princeton Univ. Press, Princeton, N.J., 1941.

[3] H. B. CuRry and R. Feys, Combinatory logic, North-Holland, Amsterdam, 1958.

[4] M. H. A. Newman, On theories with a combinatorial definition of "equivalence", Annals of mathematics, vol. 43 (1942), pp. 223-243.

[5] J. B. Rosser, Review of "A new proof of the Church-Rosser theorem," this JouRnal, vol. 21 (1956), p. 377.

[6] D. E. SCHROER, The Church-Rosser theorem, Ph.D. thesis, University of Illinois, Urbana, Ill., 1965.

UNIVERSITY OR BRISTOL 